\title{
QUALITY OF LIFE IN ELDERLY PATIENTS
}

3 MONTHS AFTER ELECTIVE SURGERY

SÃO JOÃO

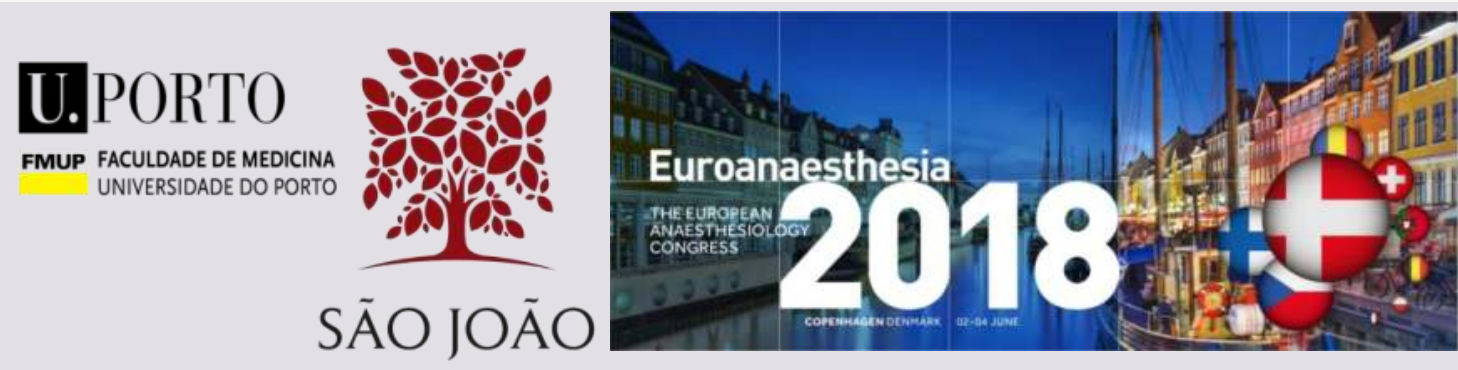

'Sarmento e Castro, J.| 1 Peixoto, A.R. | 1 Teles, A.R. |' ${ }^{1}$ Gomes Campos, M. | ${ }^{1}$ L. G. Casimiro |1,2Abelha, F.

${ }^{1}$ Dept of Anaesthesiology, Centro Hospitalar de São João, Porto - Portugal

${ }^{2}$ Department of Anaesthesiology, Hospital de São João; Faculdade de Medicina Universidade do Porto, Porto - Portugal

\section{BACKGROUND AND GOAL OF STUDY}

Health related quality of life has become a valid indicator of intervention outcomes.

The aim of this study was to evaluate quality of life in elderly patients 3 months after elective surgery (T90).

\section{MATERIAL AND METHODS}

\section{Observational prospective study}

Inclusion Criteria: Patients aged> 60 years, submitted to elective from May to July 2017 were included.

Exclusion criteria: age < 60 years old; inability to give informed consent; patients admitted in the ICU after surgery.

Health-related Quality of Life (Euro QOL 5 dimensions - EQ5D)

- Measured Quality of life (QOL) before (T0) and 3 months after surgery (T90). Poor Quality of Life (PQL) was defined as having problems in any of the 5 dimensions of EQ5D.

- EuroQol-visual analogue scale (EQ-VAS) measured at T90. Clinical Frailty Scale (CFS): evaluated at T0 and T90.

- Vulnerability if score $\geq 5$.

Quality of Recovery (QoR15):

- Evaluated before (T0) and 24h (T1) after surgery.

WHODAS (WHO Disability Assessment Schedule):

- Evaluated before (T0), 30 days (T30) and 3 months (T90) after surgery. Disability: considered to be present (DP) when WHODAS score was $\geq 25 \%$.

The Lawton Instrumental Activities of Daily Living scale (IADL): assessed independent living skills.

Charlson Comorbidity Index (CCI) quantified patient comorbidities POSSUM (Physiological and Operative Severity Score for the Enumeration of Mortality and Morbidity) was also evaluated Statistical analysis: The Chi-square, Fisher's exact or MannWhitney U tests were used for comparisons.

\section{RESULTS AND DISCUSSION}

Total of included patients: 233

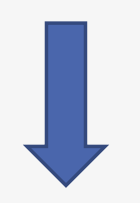

177 presented PQL at T90

Table 1 - Demographic and perioperative outcomes.

\begin{tabular}{|c|c|c|c|c|}
\hline \multicolumn{2}{|c|}{ Variable } & $\begin{array}{c}P Q L \\
(n=177)\end{array}$ & $\begin{array}{l}\text { Without PQL } \\
(n=56)\end{array}$ & $p$ value \\
\hline \multicolumn{2}{|c|}{$\begin{array}{c}\text { Age }, \\
\text { years, median [P25- P75] }\end{array}$} & 71 [65-78.5] & 67 [63-73.75] & $\mathrm{p}<0.001^{\mathrm{a}}$ \\
\hline \multirow{4}{*}{$\begin{array}{c}\text { Type of } \\
\text { Anaesthesia }\end{array}$} & General & 98 (55\%) & $40(71 \%)$ & \multirow{4}{*}{$p=0,165$} \\
\hline & Regional & $42(24 \%)$ & $10(18 \%)$ & \\
\hline & Combined & $27(15 \%)$ & $5(9 \%)$ & \\
\hline & $\begin{array}{l}\text { Sedation/ } \\
\text { Analgesia }\end{array}$ & $10(6 \%)$ & $1(2 \%)$ & \\
\hline \multirow{2}{*}{ ASA } & I/ II & $93(53 \%)$ & $43(77 \%)$ & \multirow{2}{*}{$p=0.001^{a}$} \\
\hline & III / IV & $84(47 \%)$ & $13(23 \%)$ & \\
\hline \multicolumn{2}{|c|}{ Chronic kidney disease } & $20(11 \%)$ & 0 & $p=0.009 a$ \\
\hline \multicolumn{2}{|c|}{ Stroke history } & $15(8 \%)$ & 0 & $p=0.025^{b}$ \\
\hline \multicolumn{2}{|c|}{ Smoking history } & $29(16 \%)$ & $3(54 \%)$ & $p=0.04^{b}$ \\
\hline \multicolumn{2}{|c|}{$\begin{array}{l}\text { POSSUM total score, } \\
\text { median [P25-P75] }\end{array}$} & 27 [24-30] & 25 [23-28] & $p=0.007$ \\
\hline \multicolumn{2}{|c|}{$\begin{array}{l}\text { Hospital length of stay, } \\
\text { days, median [P25- P75] }\end{array}$} & $6[2.5-13]$ & $2[2-6]$ & $p=0.048^{c}$ \\
\hline \multicolumn{2}{|c|}{$\begin{array}{l}\text { EQ-VAS at T90, } \\
\text { median, [P25-P75] }\end{array}$} & 65 [42-80] & 80 [63-95] & $<0.001^{c}$ \\
\hline
\end{tabular}

Legend: a -Qui-squared test; $\mathbf{b}-$ Fisher test; $\mathbf{c}-$ Mann-Whitney test; PQL - patients with poor quality of life 3 months after surgery; WHODAS - World Health Organization Disability Assessment Schedule; VB- Vulnerability; EQ 5D- Euro quality of life 5 dimensions; TO- before surgery;T1-24h after surgery T30- 1 month after surgery; T90- 3months after surgery;
Table 2 - Preoperative EQ-5D problems in each dimension.

\begin{tabular}{|c|c|c|c|}
\hline Preoperative EQ-5D & $\begin{array}{c}\text { PQL } \\
(\mathrm{n}=177)\end{array}$ & $\begin{array}{c}\text { Without PQL } \\
(\mathrm{n}=56)\end{array}$ & p value \\
\hline Mobility & $115(65 \%)$ & $8(14 \%)$ & \\
\hline Self-care & $76(43 \%)$ & $4(7 \%)$ & \multirow{2}{*}{$\mathbf{0 . 0 0 1}^{\mathrm{a}}$} \\
\hline Usual Activities & $96(54 \%)$ & $7(13 \%)$ & \\
\hline Pain & $113(64 \%)$ & $12(21 \%)$ & \\
\hline Anxiety & $143(81 \%)$ & $28(50 \%)$ & \\
\hline
\end{tabular}

\begin{tabular}{|c|c|c|c|}
\hline $\begin{array}{l}\text { Table 3: } \\
\text { Preoperative scores. }\end{array}$ & $\begin{array}{c}\text { PQL } \\
(n=177)\end{array}$ & $\begin{array}{c}\text { Without } \\
\text { PQL(n=56) }\end{array}$ & p Value \\
\hline \multicolumn{4}{|l|}{ Vulnerability (CFS) } \\
\hline TO & $86(49 \%)$ & $6(11 \%)$ & $p<0.001^{a}$ \\
\hline \multicolumn{4}{|l|}{ Disability (WHODAS) } \\
\hline TO & $50(28 \%)$ & $3(5 \%)$ & $\mathrm{p}<0.001^{\mathrm{a}}$ \\
\hline \multicolumn{4}{|l|}{ Lawton (IADL) } \\
\hline T0 median, [P25-75] & $5.89[4-8]$ & $7.16[7-8]$ & $p<0.001^{c}$ \\
\hline \multicolumn{4}{|l|}{ Charlson (CCI) } \\
\hline T0, median, [P25-75] & $6[4-10]$ & 4 [3-7] & $p<0.001^{c}$ \\
\hline
\end{tabular}

Table 4 - Quality of recovery according to QOR-15.

\begin{tabular}{|c|c|c|c|}
\hline QoR-15 & PQL $(\mathrm{n=82})$ & $\begin{array}{c}\text { Without PQL } \\
(\mathrm{n}=153)\end{array}$ & $\mathrm{p}$ value \\
\hline $\begin{array}{c}\text { T0, } \\
\text { median [P25-P75] }\end{array}$ & $123[105-138]$ & $138[129-144]$ & $\mathbf{p = 0 . 0 0 8 ^ { c }}$ \\
\hline $\begin{array}{c}\text { T1, } \\
\text { median [P25- P75] }\end{array}$ & $108[85-130]$ & $115[107-128.8]$ & $\mathrm{p}=0.613^{c}$ \\
\hline
\end{tabular}

\section{CONCLUSIONS}

Our study showed a high incidence of PQL at T90 in elderly patients. PQL patients were more frail and disable and had worse health status before surgery. At 3 months follow-up they still have poor quality of life. 